\title{
Profiles
}

\section{Xu Su, China CDC's Chief Expert of Radiation Protection}

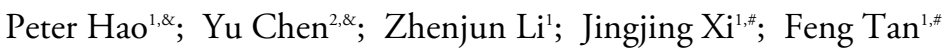

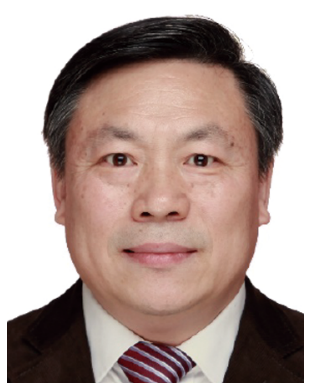

$\mathrm{Xu} \mathrm{Su}$ is the major leader of radiation protection in China and has been honored and awarded several times, has published more than 280 research papers, and has been the Director of the National Institute for Radiological Protection (NIRP) of China CDC and the Professor and Executive Director of the Chinese Center for Medical Response to Radiation Emergency from 2002 to 2017. Having been born in 1959 and, following the restoration of the National Unified Examination for Admissions to General Universities and Colleges, testing into Lanzhou University with high honors in 1977 to pursue studies in nuclear physics, $\mathrm{Xu}$ Su's entire career has been closely linked to radiological health. Following a desire to engage in radiation medicine teaching and scientific research, Xu Su then applied to the School of Public Health at Norman Bethune Medical University in Jilin Province, but due to external factors, his pursuit resulted in work in the field of nuclear radiation physics and radiation dosimetry instead of radiation medicine.

To change this situation, $\mathrm{Xu} \mathrm{Su}$ participated in the National Entrance Examination for Postgraduates in 1986 and was admitted to Norman Bethune University for radiation toxicology and was able to continue at the university to pursue a $\mathrm{PhD}$ in radiation medicine in 1993. His $\mathrm{PhD}$ supervisor was Shuzheng Liu, the president of the university and one of the most famous radiobiologists in the world, whose mentorship and instruction critically influenced Xu Su's career directions that involved researching the biological effects of low dose radiation at Toho University and at the National Institute of Radiological Sciences in Japan. Shuzheng Liu had mentioned to Xu Su that many internationally renowned radiobiologists have a background in nuclear physics, and that his own educational background will be helpful to his career development in radiobiology. Xu Su took this advice seriously, and developed into a productive researcher that was promoted to research professor in 1998.

In 1999, he was transferred to the Laboratory of Industrial Hygiene of the Ministry of Health via the talent introduction program and organized and established the Department of Radiation Biology. After China CDC was founded in 2002, the Laboratory of Industrial Hygiene of the Ministry of Health was merged into China CDC and changed its name to its current form, NIRP. During his 15 years in NIRP, Xu Su was devoted to the disciplined construction of NIRP, and with his impetus and effort, NIRP obtained approval to confer PhD and master degrees in radiation medicine. In addition, he established many subdiscipline departments and research laboratories in NIRP, such as Radiobiology, Radiation Epidemiology, Radiotoxicology, Radiation Chemistry, Radioecology, Radiation Protection, etc. In addition to his 280 published research papers, Xu Su has presided over and undertaken 26 national and ministry-level scientific research projects, edited 15 monographs, and has supervised more than 30 postdoctoral, doctoral, and postgraduate students. Since 1996, Xu Su has been awarded 6 national and provinciallevel science and technology achievement awards. He has also been included in the first wave of New Century Ten Million Talents Project National Candidate selected by the 7 Ministries of Chinese Government in 2004, recognized as the Outstanding Mid-Aged Expert of the Ministry of Health in 2004, granted Special Government Allowances approved by the Ministry of Personnel in 2005, and awarded the Award for Outstanding Contribution to Public Health and Preventive Medicine by the China Preventive Medicine Association in 2008, "Contribution Award for Radiation Research" by Asia Association for Radiation Research in 2009, and the $13^{\text {th }}$ Wu Jieping- Paul Janssen Medical \& Pharmaceutical Research Award in 2012.

As the Director of NIRP of China CDC, Xu Su led the scientific and technical personnel to conduct a large number of investigations and studies, discovered weak links in the country's radiation protection, and provided recommendations to address these issues. Under his active promotion and effort, many major projects, including the "National Medical Radiation Protection Monitoring," "National Food and Drinking Water Radiation Monitoring," "National Occupational Health Monitoring and Occupational Disease Monitoring for Radiation Workers," and 
"National Examination of Technical Ability of Radiological Health Institutions" have been successively launched, which has positively contributed to protect the health rights of workers and the public.

$\mathrm{Xu} \mathrm{Su}$ attaches great importance to international cooperation and exchanges. As chairman of the conferences, he has held many international conferences in China, such as the "International Seminar on Health Emergency in Nuclear Accidents in 2009," " $3^{\text {rd }}$ Asian Congress of Radiation Research in 2013," "First Global Chinese Radiation Research Conference in 2011," "Second Global Chinese Radiation Research Conference in 2014," and "Third Global Chinese Radiation Research Conference in 2018”. During the first Asian Congress of Radiation Research in Japan in 2005, Xu Su was elected vice-president of the Asian Radiation Research Association, and by 2013, he was elected president. From 2001 to 2014, he also participated in several meetings of the United Nations Scientific Committee on the Effects of Atomic Radiation as a member of the Chinese delegation.

Since 2002, Xu Su has organized, guided, and participated in the emergency management of many radiation emergencies. Especially during the Fukushima Daiichi Nuclear Power Plant accident in Japan in 2011 when China's public expressed panic, Xu Su carried out more than 40 information releases, expert interpretations, and responses to public questions on CCTV and other mainstream media to effectively eliminate public panic. Especially on March 17, 2011, when the public began panic buying iodized salt wrongfully believing that it could prevent absorption of radioactive iodine and that there would be a shortage of iodized salt, Xu Su appeared on CCTV and provided a detailed and vivid explanation to calm the public and resolve the incident.

$\mathrm{Xu} \mathrm{Su}$ is now working to improve China's radiation protection capabilities as the chief expert in radiation protection at China CDC.

doi: $10.46234 / \mathrm{ccdcw} 2020.148$

\# Corresponding authors: Jingjing Xi, xijj@chinacdc.cn; Feng Tan, tanfeng@chinacdc.cn.

${ }^{1}$ Chinese Center for Disease Control and Prevention, Beijing, China; ${ }^{2}$ National Institute of Environmental Health, Chinese Center for Disease Control and Prevention, Beijing, China.

\& Joint first authors.

Submitted: July 07, 2020; Accepted: July 14, 2020 